\title{
Efectos del cambio climático sobre la distribución de los alcornocales españoles. Una aproximación fitoclimática para la futura gestión
}

A. Fernández-Cancio ${ }^{1, *}$, R. Sánchez-Salguero ${ }^{1,3}$, P.M. Gil ${ }^{1}$, E. Manrique Menéndez², R. Fernádez Fernández , R.M. Navarro-Cerrillo ${ }^{3}$

(1) C.I.F.O.R.-I.N.I.A.-Crta. de La Coruña Km 7.5-28040 Madrid

(2) Universidad Politécnica de Madrid. EUIT Forestal, Dpto. Ciencias Básicas aplicadas a la Ingeniería Forestal. 29040, Madrid. España.

(3) Depto. Ingeniería Forestal, ETS Ing. Agrónomos y de Montes. Univ. Córdoba. Campus de Rabanales, Crta. IV, km. 396, 14071 Córdoba. España.

${ }^{*}$ Autor de correspondencia: A. Fernández-Cancio [fernand@inia.es]

> Recibido el 15 de octubre de 2012, aceptado el 30 de noviembre de 2012.

Fernández-Cancio, A., Sánchez-Salguero, R., Gil, P.M., Manrique Menéndez, E., Fernández Fernández, R., Navarro-Cerrillo, R.M. (2012). Efectos del cambio climático sobre la distribución de los alcornocales españoles. Una aproximación fitoclimática para la futura gestión. Ecosistemas 21(3):50-62. Doi.: 10.7818/ECOS.2012.21-3.07

Este trabajo presenta una contribución a la evaluación de los posibles efectos futuros del cambio climático en la distribución de los alcornocales (Quercus suber L.) en España, y sugiere algunas medidas alternativas de gestión. Para este objetivo se realizó una aproximación fitoclimática en función de 39 variables (periodo 1940-2005) para definir la idoneidad actual de la especie y aplicarlo en una proyección a 2050 según cuatro escenarios de cambio climático, evaluando la futura idoneidad del territorio. La diagnosis fitoclimática se realizó siguiendo la metodología de FernándezCancio y colaboradores (2004). El resultado de esta diagnosis fue la identificación de 15 grupos fitoclimáticos para las condiciones climáticas actuales, cuya distribución se alteraría principalmente por un aumento de la temperatura media y una disminución en las precipitaciones de primavera y otoño. La reducción del área de distribución del alcornoque, independientemente del escenario, resultó ser muy relevante en la mitad sur (Andalucía) y en la zona costera del noreste (Cataluña y Levante), donde la especie desaparecería prácticamente como formaciones de alcornocal, y en una parte muy importante del territorio extremeño. Los resultados obtenidos ponen de manifiesto una posible idoneidad futura de los alcornocales en el noroeste de España (Galicia y Cornisa Cantábrica) como áreas de refugio. Sin embargo, Quercus suber encontrará un serio limitante para una potencial migración en la disponibilidad reducida de áreas geográficas con edafología adecuada, salvo en Portugal y en el corredor de ExtremaduraSalamanca. Según esta simulación podrían requerirse medidas alternativas de gestión para la mitigación de los efectos del cambio climático en los alcornocales españoles.

Palabras clave: Quercus suber, fitoclimatología, vulnerabilidad, decaimiento forestal

Fernández-Cancio, A., Sánchez-Salguero, R., Gil, P.M., Manrique Menéndez, E., Fernández Fernández, R., Navarro-Cerrillo, R.M. (2012). Climatic change effects on the distribution of cork oak Spanish forests. A phytoclimatic approach for the future management. Ecosistemas 21(3):50-62. Doi.: 10.7818/ECOS.2012.21-3.07

This manuscript presents a contribution in the evaluation of possible future climatic change effects on the distribution of cork oak forests (Quercus suber L.) in Spain, and suggests new management alternatives. To this aim, we used a phytoclimatic approach using 39 variables (time period 19402005 ) to define the current suitability and apply a projection to 2050 under four climatic change scenarios to define the future species suitability. The phytoclimatic diagnosis followed Fernández-Cancio et al. (2004). This diagnosis identified 15 phytoclimatic groups for current climatic conditions, which could shift their distribution as a result of an increase in average temperature and a decrease in spring and autumn precipitation. The decrease in cork oak distribution, regardless of the climate scenario, will be very relevant in southern Spain (Andalusia) and the northeastern coastal area (Catalonia and Levante), where cork oak dense forests may disappear, as well as in the Extremadura region. The results suggest that new suitable areas might appear in northwestern Spain (Galicia and Cantabria) as refuge areas. However, Quercus suber will find a serious limitation to migrate as a results of the scarce availability of adequate soil in the potential geographic areas, except in Portugal, Extremadura and Salamanca regions. According with these simulations, new management alternatives to mitigate the effects of climate change in the Spanish cork oak forests will be required.

Keywords: Quercus suber, phytoclimatology, vulnerability, forest decline

\section{Introducción}

Las estrategias para la conservación de ecosistemas necesitan anticiparse al cambio climático. A escala global dicho cambio ha sido ampliamente debatido en el ámbito científico e institucional, y hoy en día casi nadie cuestiona que se esté produciendo un cambio significativo de las condiciones climáticas, con un incremento de temperatura de $0.7^{\circ} \mathrm{C}$ en el siglo pasado, y un incremento previsto por encima de $2.6^{\circ} \mathrm{C}$ durante el presente siglo (IPCC 2007). El cambio climático tendrá, por tanto, un impacto muy importante sobre muchas especies y comunidades vegetales en todos los ecosistemas del mundo. Algunas especies, como el alcornoque (Quercus 
suber L.) pueden ver alterados sus patrones de distribución actual en la España peninsular debido a este aumento de las temperaturas (Ribalaygua et al. 1998; Benito et al. 2008). Los gestores y propietarios de monte alcornocal se pueden enfrentar al dilema de cuáles son las actuaciones más recomendables para su conservación (Montero y Cañellas 2003; Bronchalo González et al. 2011).

Los procesos de decaimiento y mortalidad relacionados con las nuevas condiciones y variabilidad climáticas son un fenómeno recurrente en numerosos ecosistemas forestales a escala global y regional (Peñuelas et al 2001; Camarero et al. 2004; Allen et al. 2010), y en particular en el Mediterráneo, donde se prevé un aumento de la aridez (Lloret 2012; Martínez-Vilalta et al. 2012). En consecuencia, la predicción del comportamiento de la vegetación a medio o largo plazo, en respuesta a cambios climáticos inducidos por el calentamiento global, es uno de los desafíos científicos más importantes en la actualidad (Petit et al. 2005).

Desde principios del siglo XX y a lo largo de toda Europa, las especies de Quercus han mostrado diversos episodios de estrés que en ocasiones han dado lugar al decaimiento y muerte del arbolado (Brasier 1996; Fernández-Cancio et al. 2004; Dobyshev et al. 2007). En España parecen haber sufrido de forma especial una perturbación a gran escala según se observa en la Red Nacional de Seguimiento de Daños de Nivel I (Montoya y López Arias 1997; Carnicer et al. 2011) y, dada su importancia ecológica y económica, el estudio de este problema parece de especial relevancia (Allué-Andrade 1995; Fernández-Cancio et al. 2004). Estas especies presentan un área de distribución muy amplia y con cierta plasticidad ecológica, es decir, se adaptan razonablemente bien a diferentes climas y en menor medida a diferentes suelos, y no son muy exigentes, por lo que los efectos del cambio climático no van a comprometer su supervivencia como especie (Gil 2009; Linder et al. 2010; Felicísimo et al. 2010). Sin embargo, sus representaciones actuales más importantes en general pueden verse reducidas (Linder et al. 2010; Felicísimo et al. 2010). Por otro lado, las especies de Quercus se verán sometidas a la competencia con otras especies y al incremento de la incidencia de perturbaciones como incendios forestales, plagas y enfermedades (Carnicer et al. 2011). Todo ello hará que la conservación de estos ecosistemas se vuelva más compleja y costosa en un contexto de cambio climático (Felicísimo et al. 2010; García-López y Allué-Camacho 2011), a menos que se comiencen a desarrollar y aplicar medidas específicas para mitigar y adaptar la especie a los efectos del cambio climático.

Como disciplina para desarrollar estas medidas de mitigación, la fitoclimatología se presenta como un conjunto de modelos numérico-conceptuales que permiten asociar los límites de existencia y áreas de distribución de las especies a determinadas variables ambientales. Según numerosos trabajos previos (Allué-Andrade 1990; Allué-Andrade y Fernández-Cancio 1993; Fernández-Cancio et al. 2004; Gil 2009; Gonzalo Jiménez 2010), es habitual que los parámetros fitoclimáticos estén correlacionados con los factores limitantes de la distribución geográfica y altitudinal de las especies, condicionándolas según sus necesidades y afinidades ecológicas (Box, 1995; Guisan y Zimmermann, 2000; Araujo y Guisan, 2006). Cualquier modificación sostenida en los parámetros fitoclimáticos tiene, por tanto, respuesta indirecta pero observable en la distribución y desplazamiento de la vegetación, y esto se puede simular en un escenario de cambio climático (IPCC 2007; García-López y Allué-Camacho 2011). Cuando además aparecen procesos de mortalidad y cambios en la estructura del paisaje el cambio climático se convierte ya en un cambio fitoclimático o bioclimático en un sentido más general, afectando a plantas, animales y seres humanos (Allué-Andrade y Fernández-Cancio 1993).

En la definición de escenarios futuros simulados es imprescindible el conocimiento de la autoecología actual del alcornoque, que ha sido ampliamente estudiada por Elena Rossello et al. (1990), y sintetizada por Elena Rossello (2004), así como por Maldonado et al. (2002); Fernández-Cancio et al. (2004); García-López y AlluéCamacho (2009), y Gil (2009), definiendo en España un hábitat para la especie más o menos amplio según los autores. En este trabajo se ha intentado dar respuesta a los posibles efectos del cambio climático sobre la distribución actual de Quercus suber en España. Se ha estudiado la simulación de cuatro escenarios climáticos probables contenidos dentro de las proyecciones oficiales de cambio climático para el siglo XXI (AEMET 2010), para estimar la posible distribución futura del alcornoque basada en estudios previos de fitoclimatología, autoecología paramétrica y escenarios de cambio climático (Fernández-Cancio et al. 2004; IPCC 2007; AEMET 2010). Se debe tener en cuenta que estamos ante simulaciones que no contemplan aspectos como la inercia de los ecosistemas, las adaptaciones ambientales mediante modificaciones fisiológicas y biotípicas, así como la selección genética de los individuos y de las poblaciones mejor adaptadas. Cuando se habla de factores limitantes se hace referencia a los parámetros medidos por las estaciones meteorológicas o las relaciones entre ellos que se suponen asociadas a los verdaderos factores limitantes desde un punto de vista correlacional, no causal, y que establecen límites observables en la biogeografía, de modo que la sectorizan de forma coherente con un sentido fitológico (Allué 1990; Rivas Martínez 2007).

\section{Material y Métodos}

\section{Ámbito de existencia fitoclimática de Quercus suber}

Los alcornocales constituyen una de las comunidades forestales más características de la "Iberia seca" (Fig. 1). La ecología de los alcornocales ha sido ampliamente estudiada (Blanco Castro et al. 1997; Elena Roselló 2004; Ruiz de la Torre 2006). El bioclima general de los bosques de alcornocal está condicionado por el gradiente altitudinal (entre 0 y $1200 \mathrm{~m}$.), unas precipitaciones supe-

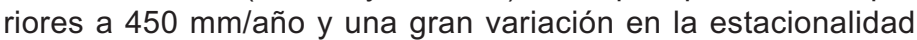
pluviométrica oeste-este en España. El alcornoque sólo aparece sobre sustratos ácidos que originen suelos con buen drenaje y buena aireación. Es una especie netamente calcífuga, no aparece sobre terrenos calizos a menos que se encuentren descarbonatados (Blanco Castro et al. 1997).

Fitoclimáticamente se puede asumir que en España existen alcornocales asociados a diferentes comportamientos de las variables fitoclimáticas estudiadas (Pereira 2002; Fernández-Cancio et al. 2004; Gil 2009). Para poder realizar este análisis es necesario conocer los límites fitoclimáticos de existencia de la especie y también establecer un conjunto amplio de variables fitoclimáticas desde el cual el estudio de escenarios de cambio climático futuros sea posible.

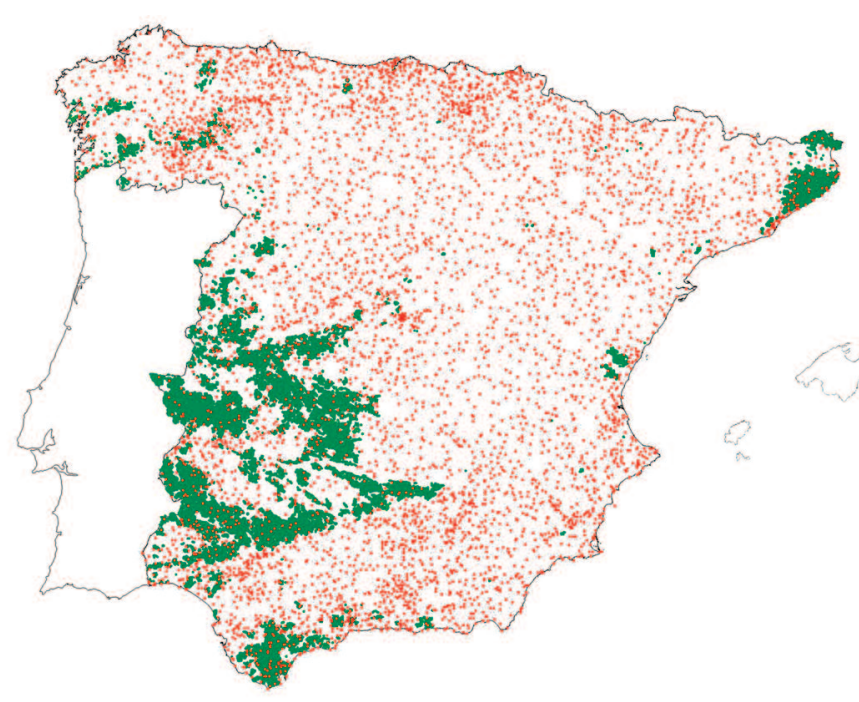

Figura 1. Distribución actual del alcornoque (Quercus suber L.) (verde) y estaciones meteorológicas utilizadas en España (rojo) (MMA 1997 y AEMET 2010). 


\section{Datos corológicos y climáticos}

A partir de la base de datos de parcelas de muestreo correspondientes al II Inventario Forestal Nacional, IFN2 (Villanueva 1990; MMA 1997), se seleccionaron todos los puntos con presencia natural de Quercus suber como especie principal de la formación forestal, cotejándolos con diversas fuentes de información (Rivas Martínez 1987, 2007; Allué Andrade 1990; Mapa Forestal de España de Ruiz de la Torre 1990-1992; Base de datos de DGB, 2003; Bases INM, Fernández Cancio et al. 2001) para abarcar con la mayor precisión posible su área de distribución actual. Por otro lado, los datos climáticos utilizados proceden de 5008 estaciones meteorológicas (AEMET 2010) a partir de los cuales se calcularon las distintas variables fitoclimáticas (Fernández-Cancio et al. 2004; Gil 2009) (Fig. 1). Esta información climática se fundamentó en el cómputo de valores medios normalizados a lo largo del periodo 1940-2005, de manera que cada estación meteorológica revela el tipo de clima correspondiente a un lugar determinado durante un intervalo temporal de 65 años.
Con la información corológica de Q. suber y las estaciones meteorológicas se organizó una red de información fitoclimática. La elaboración de esta red consiste en la localización y asignación de estaciones meteorológicas próximas a las coordenadas geográficas $(\mathrm{X}, \mathrm{Y}, \mathrm{Z})$ de cada parcela seleccionada del IFN2 en un radio inferior a $2 \mathrm{~km}$ de distancia del punto corológico y con una diferencia de $\pm 100 \mathrm{~m}$ de altitud. De esta manera cada punto de presencia de las cartografías corológicas elaboradas tuvo asociado un número determinado de estaciones meteorológicas incluidas en el área de distribución de la especie (Fernández-Cancio et al. 2004; Gil Hernández 2009). La compatibilidad edáfica se ha establecido considerando sólo las estaciones que están asociadas a series potenciales de vegetación de carácter ácido.

A partir de esta red se calcularon los factores fitoclimáticos de Allué-Andrade $(1990,1995)$ y bioclimáticos de Rivas-Martínez (2007) y se complementó con la generación de una serie de nuevos parámetros climáticos empleados en estudios fitoclimáticos previos siguiendo la metodología de Fernández-Cancio et al. (2004,

Tabla 1. Parámetros utilizados en el estudio de viabilidad fitoclimática del alcornoque ante escenarios de cambio climático. (Fernández Cancio et al. 2004).

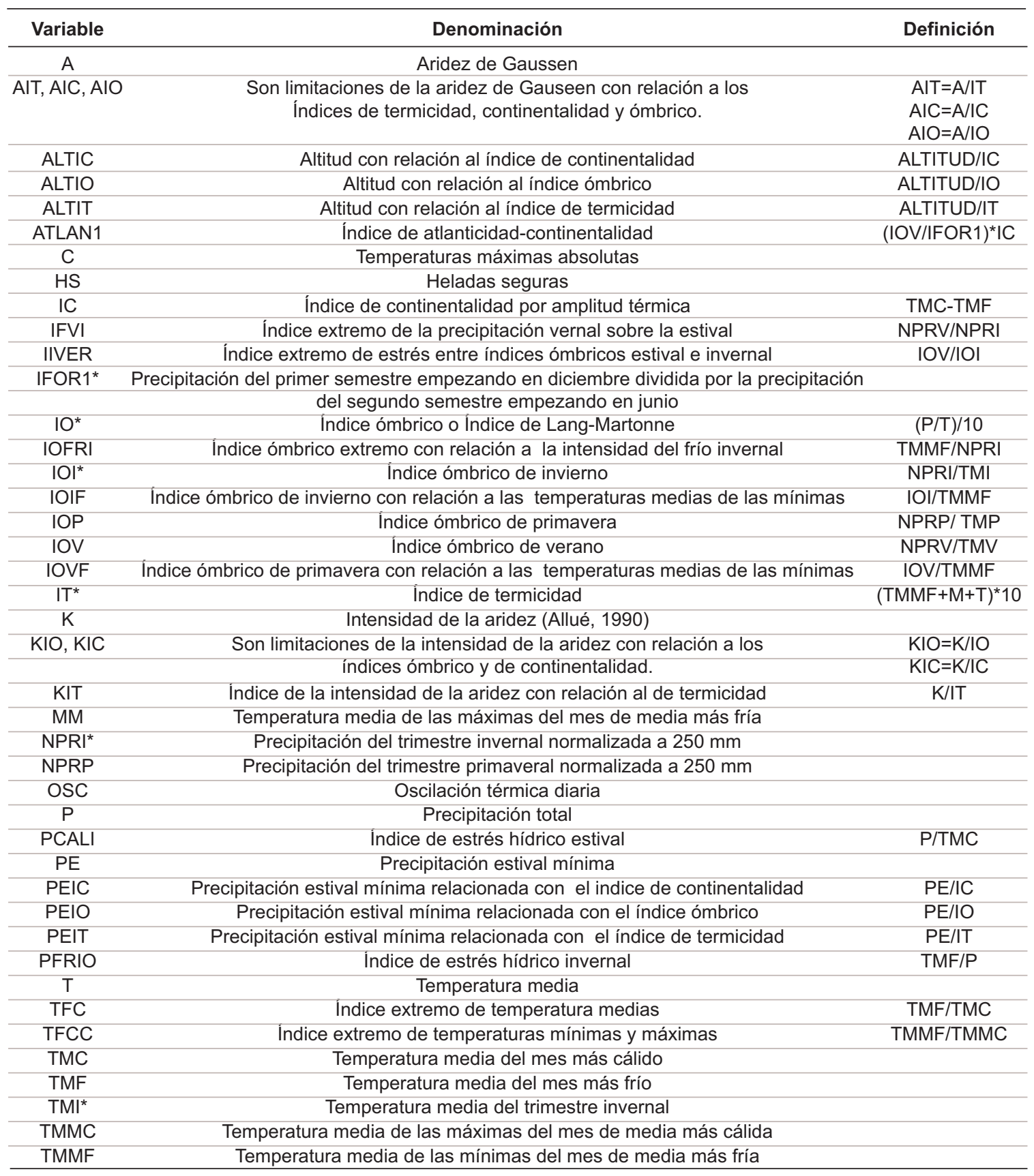

*No incluida en el modelo, sólo para explicación de otros parámetros. Para mayor detalle sobre las variables y metodología ver Fernández-Cancio et al. (2004). 
2011), Rivas Martínez (2007) y Gil Hernández (2009) (Tabla 1). Los parámetros fitoclimáticos se seleccionaron por su capacidad discriminante en el análisis de adecuación fitoclimática de la vegetación, con especial atención hacia aquellos que resaltaban las características del alcornocal. Este proceso de selección de variables consistió en una depuración previa de datos meteorológicos y posteriormente en un análisis cluster por el método de Ward y la distancia Euclídea; además de un análisis discriminante para perfeccionar y concluir la clasificación en diferentes grupos fitoclímaticos de alcornocal en función de 39 parámetros (Pereira y Fernández-Cancio 2001; Pereira, 2002; Fernández-Cancio et al. 2004; Gil 2009) (Tabla 1).

\section{Escenarios de cambio climático}

Los escenarios que se han aplicado en este trabajo están basados en los modelos de circulación general de la atmósfera (GCM) para España (AEMET 2010), pero las hipótesis de trabajo (escenarios) que se han empleado en el estudio del alcornocal, según los resultados de su distribución fitoclimática, se han limitado para el horizonte más fiable del año 2050 (Brunet et al. 2009). Asímismo se ha fijado una tendencia de un aumento máximo de $2^{\circ} \mathrm{C}$ en la temperatura y un aumento y una disminución general de 10-15\% en la precipitación, con variaciones estacionales basadas en los plausibles efectos observados en diversos trabajos (Andreu et al. 2007; García-López y Allué-Camacho 2011). Se consideraron cuatro escenarios posibles dentro de los márgenes de las proyecciones oficiales de la AEMET (proyecto ENSEMBLES 2009):

- Escenario $\mathrm{A}_{1}$ : aumento de temperatura media anual de $2^{\circ} \mathrm{C}$ y precipitación anual igual a la del período 1900-2005 en toda el área peninsular.

- Escenario $\mathrm{A}_{2}$ : aumento de $2^{\circ} \mathrm{C}$ y disminución general de precipitaciones de un 15\% respecto al periodo 1900-2005 en toda el área peninsular.

- Escenario $\mathrm{A}_{3}$ : aumento de $2^{\circ} \mathrm{C}$ y aumento de precipitación estacional según cuencas hidrográficas (Gandullo et al 2008). Así, durante el invierno, las cuencas Catalana, Ebro, Levante y Duero aumentarían su precipitación un $10 \%$, las cuencas del Tajo, Guadiana y Guadalquivir un $30-40 \%$, y las del Sur y Levante un $25 \%$. Las cuencas Cantábricas no se modificarían. En primavera las cuencas Cantábricas, Duero, Tajo, Guadiana, Sur y Guadalquivir aumentarían entre un $0-10 \%$, mientras que la Catalana, Ebro y Levante se mostrarían sin cambios. Durante el verano sólo las cuencas Cantábricas, Tajo y Guadiana aumentarían su precipitación un 10\%. Por último, durante el otoño habría una disminución pluviométrica de un $10 \%$ en las cuencas Catalana y Cantábricas.

- Escenario $\mathrm{A}_{4}$ : aumento de $2^{\circ} \mathrm{C}$ y cambio estacional de precipitación con una pérdida de un 5-7\% en la precipitación anual.

La elección de estos cuatro escenarios cubre las situaciones más favorables climáticamente dentro de las probables según los escenarios previstos (Ribalaygua et al. 1998; Nakicenovic et al. 2000; IPCC 2007; AEMET 2010). Por el momento, ningún escenario regional parece más preciso y exacto en media que los que se utilizan en el presente trabajo, pues los escenarios locales propuestos, más matizados, establecen predicciones que en su banda de confianza ya se encuentran aquí representados. Se ha considerado siempre como aumento máximo de temperatura $2^{\circ} \mathrm{C}$ en el 2050 , ya que por encima de este umbral, los modelos muestran una desestabilización climática impredecible con los datos actuales según la respuesta diferencial de los GCM existentes (Brunet et al. 2009)

\section{Resultados y Discusión}

\section{Ámbito de existencia fitoclimática de Quercus suber}

En la tabla 2 se muestran los límites fitoclimáticos de las 39 variables finalmente seleccionadas para 455 estaciones meteorológicas incluidas en el área de distribución actual de Q. suber (Fig. 2). Los resultados establecen 15 grupos fitoclimáticos (Tabla 3; Fig. 2).
Tabla 2. Límites de existencia fitoclimática de Quercus suber para cada una de las 39 variables seleccionadas, más la altitud, dando su rango y los valores obtenidos mediante percentiles al 5\% y 95\%. Ver descripción de las variables utilizadas en Tabla 1.

\begin{tabular}{|c|c|c|c|c|}
\hline \multicolumn{5}{|c|}{ Quercus suber } \\
\hline Variables & Mínimo & Máximo & $5 \%$ & $95 \%$ \\
\hline A & 0 & 5.2 & 1.22 & 4.45 \\
\hline AIC & 0 & 0.42 & 0.09 & 0.31 \\
\hline $\mathrm{AlO}$ & 0 & 18.57 & 2.64 & 15.18 \\
\hline AIT & 0 & 0.18 & 0.05 & 0.14 \\
\hline ALTIC & 0.23 & 5.68 & 0.45 & 4.18 \\
\hline ALTIO & 0.87 & 24.51 & 1.71 & 19.29 \\
\hline ALTIT & 0.1 & 3.81 & 0.18 & 2.85 \\
\hline ATLAN1 & 0 & 6.05 & 0.15 & 2.53 \\
\hline C & 34 & 49.7 & 38.7 & 47 \\
\hline $\mathrm{HS}$ & 0 & 1 & 0 & 0 \\
\hline IC & 11.4 & 23.5 & 12.9 & 20.5 \\
\hline IFVI & 0 & 1.26 & 0.05 & 0.57 \\
\hline IIVER & 0 & 0.49 & 0.02 & 0.21 \\
\hline IOFRI & -9.5 & 14 & 0.16 & 2.1 \\
\hline IOIF & -0.35 & 13.53 & 0.38 & 8.49 \\
\hline IOP & 0.24 & 1.52 & 0.32 & 0.78 \\
\hline IOV & 0 & 0.37 & 0.02 & 0.21 \\
\hline IOVF & -2.69 & 800 & 4.7 & 285 \\
\hline $\mathrm{K}$ & 0 & 0.58 & 0.02 & 0.44 \\
\hline $\mathrm{KIC}$ & 0 & 0.4 & 0.01 & 0.27 \\
\hline $\mathrm{KIO}$ & 0 & 0.24 & 0 & 0.15 \\
\hline KIT & 0 & 0.16 & 0.01 & 0.14 \\
\hline MM & 7 & 19.9 & 9.5 & 15.8 \\
\hline NPRP & 0.47 & 1.48 & 0.58 & 1.21 \\
\hline OSC & 6.1 & 17.9 & 8.6 & 14.9 \\
\hline $\mathrm{P}$ & 507 & 1448 & 542 & 1201 \\
\hline PCALI & 0.19 & 0.87 & 0.21 & 0.5 \\
\hline PE & 0 & 62 & 0 & 28 \\
\hline PEIC & 0 & 4.13 & 0 & 1.92 \\
\hline PEIO & 0 & 1.04 & 0 & 0.59 \\
\hline PEIT & 0 & 0.22 & 0 & 0.11 \\
\hline PFRIO & 0.3 & 2.1 & 0.58 & 1.69 \\
\hline $\mathrm{T}$ & 9.4 & 20.1 & 13.3 & 18.1 \\
\hline TFC & 0.18 & 0.51 & 0.22 & 0.47 \\
\hline TFCC & -0.02 & 0.33 & 0.02 & 0.25 \\
\hline TMC & 16.2 & 30.1 & 21.2 & 27.7 \\
\hline TMF & 4.3 & 12.9 & 5.3 & 11.6 \\
\hline TMMC & 22.4 & 41.4 & 27.8 & 36.8 \\
\hline TMMF & -0.7 & 9.1 & 0.6 & 7.5 \\
\hline
\end{tabular}

En la Península Ibérica se han realizado diversos trabajos sobre la fitoclimatología de Quercus suber que emplean técnicas estadísticas similares (Pereira y Fernández-Cancio 2001; Pereira 2002; Gonzálo Jiménez 2010), pero en este trabajo se incluyen nuevos parámetros fitoclimáticos (Tabla 1 y 2) (Fernández-Cancio et al. 2004; Gil 2009) que matizan y ajustan los resultados. Por ejemplo, se explica el comportamiento de alcornocales disgregados de las masas principales (Asturias y Galicia) (grupos 4 y 5) y además se identifican claramente los alcornocales extremeños-andaluces de preferencia subcontinental (grupo 7), los centromeridionales mesomediterráneos húmedos-subhúmedos (grupos 8,9 y 10) y los gaditanos termo-mesomediterráneos subhúmedos-húmedos de influencia oceánica (grupos 13, 14 y 15) (Fig. 2).

Los subtipos fitoclimáticos genuinos de Allué y los biotipos de Rivas Martínez para Q. suber incluidos en este trabajo pueden verse en mayor detalle en Allué-Andrade (1995), Maldonado et al. (2002) y Rivas Martínez (2007). Según estos trabajos, los alcorno- 


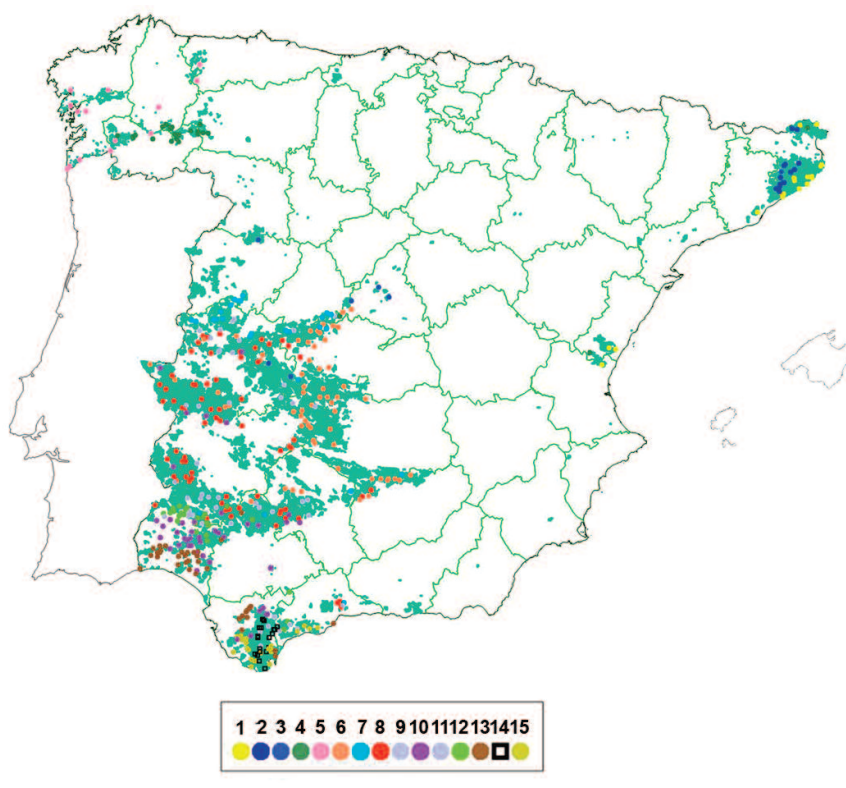

Figura 2. Grupos fitoclímáticos definidos para las estaciones meteorológicas que poseen alcornocal a menos de $2 \mathrm{~km}$. de distancia y una diferencia de altitud de \pm 100 . Los códigos numéricos para cada grupo son los mismos que los usados en la Tabla 2. cales onubo-gaditanos termomediterráneos (grupos 12 y 13) y los gaditanos termo-mesomediterráneos subhúmedos-húmedos de influencia oceánica (grupos 14 y 15) presentarían la máxima genuinidad coincidiendo parcialmente con resultados previos (Gil 2009). Los alcornocales onubense-gaditanos-malacitanos termo-mesomediterráneos subhúmedos (grupos 10 y 14) se sitúan en una posición intermedia; los alcornocales catalano-valencianos de influencia centroeuropea y Tethyca (grupos 1 y 2) se sitúan junto a los alcornocales galaico-leoneses de inviernos fríos (grupos 3 y 4). Así como los alcornocales asturianos y galaico occidentales (grupo 5) estarían en una posición más marginal.

De forma más concreta Allué y Montero (1989) aplicaron este tipo de modelos de idoneidad a los alcornocales catalanes y los resultados coinciden con los obtenidos en el grupo 1 y 2 (alcornocales catalano-valencianos de influencia centroeuropea y Tethyca) (Maldonado et al. 2002; Gil 2009).

El análisis discriminante realizó la calibración-verificación de los grupos fitoclimáticos definidos (Tabla 3 ) de forma estadísticamente significativa al $99.99 \%$, clasificando correctamente las clases en un $98 \%$ y $94 \%$ de los casos, respectivamente.

\section{Impacto del cambio climático sobre la distribución del alcor- noque en España}

La combinación de los 15 grupos fitoclimáticos definidos con los cuatro escenarios de cambio climático propuestos permite hacer

Tabla 3. Grupos de alcornocal obtenidos por clasificación jerárquica.

\begin{tabular}{|c|c|c|c|}
\hline Grupos & Número de Estaciones & $\%$ sobre el total & Grupos fitoclimáticos \\
\hline 1 & 19 & 4.17 & $\begin{array}{l}\text { Alcornocal catalán con influencia costera termo-mesomediterráneo seco- } \\
\text { subhúmedo de carácter esclerófilo ilicino Thetyco típico. }\end{array}$ \\
\hline 2 & 17 & 3.73 & $\begin{array}{l}\text { Alcornocal catalán no costero mesomediterráneo superior subhúmedo de } \\
\text { carácter esclerófilo ilicino Thetyco con tendencias nemorales. }\end{array}$ \\
\hline 3 & 7 & 1.53 & $\begin{array}{l}\text { Alcornocal de irradiación biogeográfica amplia de orla de montaña meso- } \\
\text { supramediterráneo seco-subhúmedo de carácter esclerófilo ilicino con } \\
\text { tendencias marcescentes }\end{array}$ \\
\hline 4 & 27 & 5.93 & $\begin{array}{l}\text { Alcornocal gallego orensano-lucense supramediterráneo seco-subhúmedo de } \\
\text { carácter transicional hacia la planocaducifolia con marcescencia obligada. }\end{array}$ \\
\hline 5 & 13 & 2.85 & $\begin{array}{l}\text { Alcornocal gallego colino-termocolino subhúmedo-húmedo de carácter } \\
\text { nemoral planocaducifolio con esclerofilia marginal climácica. }\end{array}$ \\
\hline 6 & 59 & 12.96 & $\begin{array}{l}\text { Alcornocal extremeño-manchego-jienense mesomediterráneo subhúmedo- } \\
\text { seco de carácter esclerófilo ilicino mediterráneo típico más árido. }\end{array}$ \\
\hline 7 & 27 & 5.93 & $\begin{array}{l}\text { Alcornocal andaluz-extremeño-gredense de orla de montaña de irradiación } \\
\text { biogeográfica amplia mesomediterráneo subhúmedo-húmedo de carácter } \\
\text { esclerófilo nemoral transicional hacia la marcescencia. }\end{array}$ \\
\hline 8 & 80 & 17.58 & $\begin{array}{l}\text { Alcornocal extremeño- andaluz de irradiación biogeográfica amplia } \\
\text { mesomediterráneo inferior seco-subhúmedo de carácter esclerófilo ilicino } \\
\text { mediterráneo típico muy árido. }\end{array}$ \\
\hline 9 & 45 & 9.89 & $\begin{array}{l}\text { Alcornocal andaluz -extremeño de irradiación biogeográfica amplia } \\
\text { mesomediterráneo inferior subhúmedo de carácter esclerófilo ilicino } \\
\text { mediterráneo típico. }\end{array}$ \\
\hline 10 & 33 & 7.25 & $\begin{array}{l}\text { Alcornocal de núcleo onubense con irradiación biogeográfica amplia termo- } \\
\text { mesomediterráneo subhúmedo-seco de carácter esclerófilo ilicino } \\
\text { mediterráneo típico más árido. }\end{array}$ \\
\hline 11 & 14 & 3.07 & $\begin{array}{l}\text { Alcornocal andaluz de orla de montaña meso-termomediterráneo húmedo de } \\
\text { carácter esclerófilo mediterráneo transicional con tendencias nemorales. }\end{array}$ \\
\hline 12 & 33 & 7.25 & $\begin{array}{l}\text { Alcornocal sevillano-onubense meso-termomediterráneo subhúmedo de } \\
\text { carácter esclerófilo ilicino mediterráneo típico. }\end{array}$ \\
\hline 13 & 37 & 8.13 & $\begin{array}{l}\text { Alcornocal gaditano-onubense termomediterráneo seco de carácter } \\
\text { esclerófilo transicional hacia el infrailicino. }\end{array}$ \\
\hline 14 & 20 & 4.39 & $\begin{array}{l}\text { Alcornocal gaditano- malagueño termomediterráneo superior subhúmedo de } \\
\text { carácter esclerófilo transicional hacia el infrailicino. }\end{array}$ \\
\hline 15 & 24 & 5.27 & $\begin{array}{l}\text { Alcornocal gaditano-malagueño termomediterráneo inferior húmedo de } \\
\text { carácter esclerófilo transicional hacia el infrailicino. }\end{array}$ \\
\hline
\end{tabular}




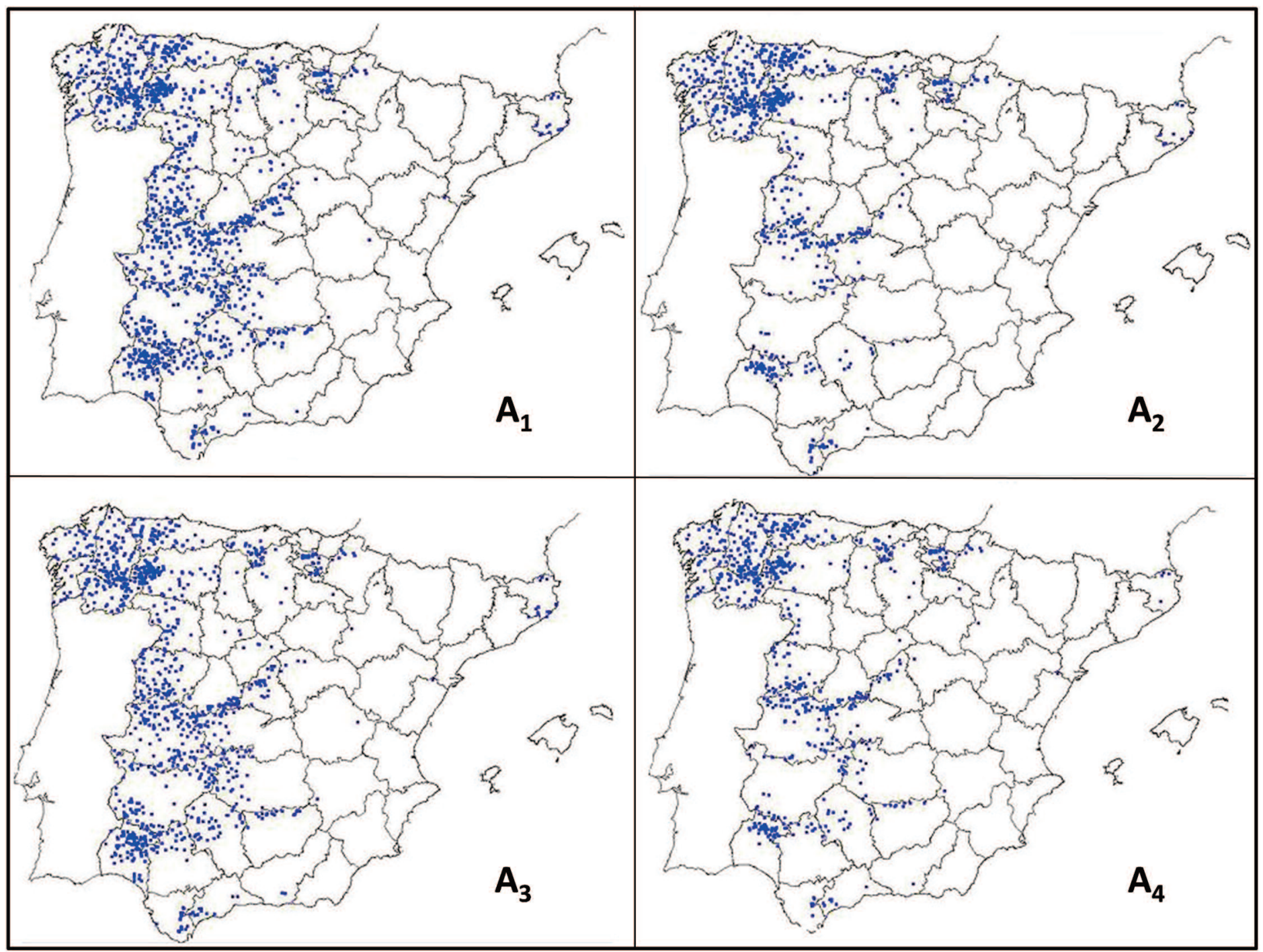

Figura 3. Impacto del cambio climático bajo los escenarios $A_{1}, A_{2}, A_{3}$ y $A_{4}$ definidos sobre la distribución de los ecosistemas de alcornoque en la Península Ibérica.

proyecciones y extraer conclusiones del impacto climático sobre la posible distribución futura de los alcornocales (Fig. 2).

\section{Escenario $A_{1}$}

Se observa un aumento de la idoneidad de carácter exclusivamente térmico y con efectos generales de mayor estrés hídrico del alcornocal hacia el Norte de la Península Ibérica siguiendo el posible pasillo migratorio occidental (Extremadura-Salamanca) (Fig. 3) y a instalarse en las zonas basales de los sistemas montañosos, en una línea coherente con el trabajo de Felicísimo et al. (2010).

En este escenario el alcornocal permanecería en algunos de sus enclaves andaluces, como Sierra Morena y el Parque Natural de los Alcornocales, que mantendrían las poblaciones actuales de la especie. Según este escenario, la zona onubense y gaditana perdería la idoneidad de la mayor parte de sus alcornocales de baja altitud (Benito et al. 2008; Felicísimo et al. 2010). En el resto de España aumentarían las zonas idóneas en Galicia, Asturias y la franja cantábrica que se convertirían en zonas de mayor estabilidad para el alcornocal en este escenario previsto. El impacto sobre los alcornocales catalanes y levantinos sería importante, ya que podrían abandonar la zona de costa (Benito et al. 2008). En Extremadura el alcornocal se podría desplazar hacia el norte abandonando las zonas más térmicas. Además, habría un importante potencial de posible expansión en Castilla-León y en las provincias de Madrid y Ávila siguiendo claramente el eje del Sistema Central (Felicísimo et al. 2010). El posible desplazamiento de la especie hacia zonas orientales estaría limitado en algunos territorios por la litología.
En resumen, los principales efectos sobre la distribución del alcornocal para este escenario serían un cambio moderado de sus áreas de distribución potencial, con las reducciones más importantes de superficie en Andalucía y Cataluña. Si el alcornocal tuviera posibilidades de migrar, podría experimentar un desplazamiento importante hacia el norte, en particular en la parte occidental de Galicia y Asturias.

Analizando la idoneidad de cada grupo fitoclimático definido en este escenario, la superficie de mayor potencialidad se desplazaría hacia el norte o el noreste (Felicísimo et al. 2010). El número de estaciones meteorológicas en las que encontramos compatibilidad con el alcornocal en este escenario es de 1109. El aumento sobre las 455 actuales se debe a la mayor densidad de estaciones presentes en las cuencas del noroeste y recorridos medios del Duero y Tajo, asociadas a la red de control hidrológico. Por tanto, deben ser tomadas como una referencia inicial relativa de pérdida o ganancia de potencialidad con respecto a la distribución actual, que no selecciona las estaciones potencialmente aptas sino sólo aquellas donde se encuentra hoy el alcornoque.

En la Figura 4 se ha representado los puntos de cada grupo fitoclimático sin efectos por este escenario y las nuevas estaciones corológicas que aparecerían en cada grupo fitoclimático según la expansión territorial del escenario $A_{1}$. En este primer escenario no se consideran los efectos del suelo, en los siguientes sí. Se puede observar la capacidad de algunos grupos para ampliar su área y las dificultades ante los cambios en la temperatura. El resultado depende en muchos casos del mayor o menor rango de sus variables, 

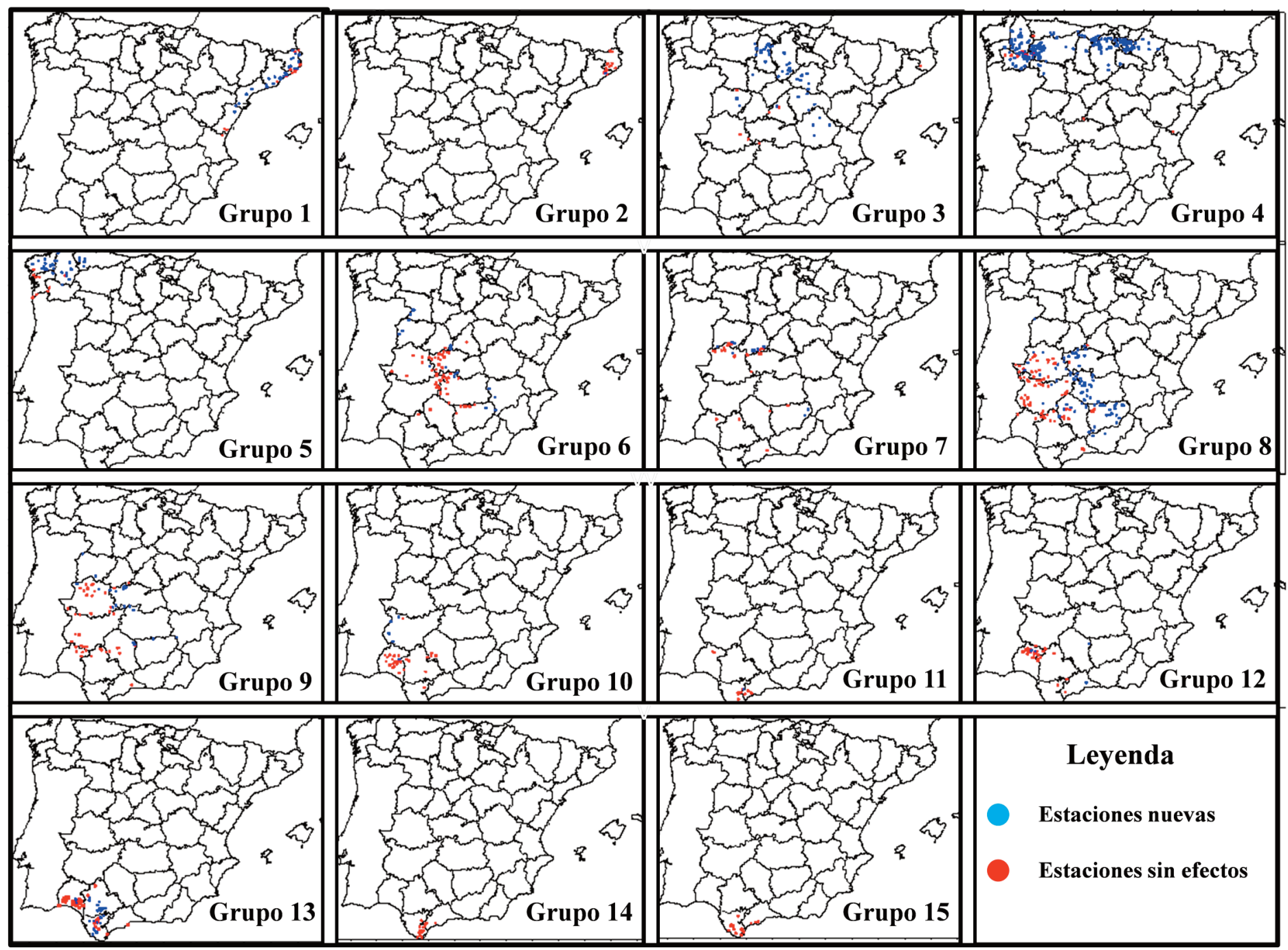

\section{Leyenda}

Estaciones nuevas

Estaciones sin efectos

Figura 4. Nuevas posiciones de cada grupo tipo fitoclimático de alcornoque (ver tabla 2) para el escenario $A_{1}$. En rojo se ha representado los puntos sin efectos del escenario y en azul la dispersión de nuevas posiciones territoriales en España.

así como de la capacidad específica del territorio en términos de homogeneidad. De los 15 grupos de alcornoque en el Escenario $A_{1}$ se expandiría la potencialidad en los grupos $1,3,4,5,8,9$ y 13. Los grupos 2, 6, 7, 10, 11 y 12 sufrirían fuertes contracciones y los grupos 14 y 15 no serían viables en este escenario (Fig. 4).

\section{Escenario $\mathrm{A}_{2}$}

Este escenario muestra como principal característica la sensibilidad del alcornoque al régimen de precipitaciones (Fig. 3). En estas condiciones se produciría una contracción general de su área ibérica y sólo aquellos enclaves que mantengan precipitaciones altas soportarían la subida térmica y la aridez inducida por la disminución de precipitación (Benito et al. 2008; Urbieta et al. 2008). El alcornoque se conservaría bien en Galicia y franja cantábrica, perdiendo sólo algunos enclaves en estas zonas, pero su área de idoneidad se vería reducida en el resto de sus poblaciones españolas, sobre todo en Extremadura y Andalucía cuyos núcleos permanentes estarían en Gredos, Parque Natural de los Alcornocales y Sierra Morena Occidental (Felicísimo et al. 2010). En Cataluña el impacto también sería importante, quedando restringido a las proximidades de los sistemas montañosos.

Este escenario es el que supone un impacto mayor sobre los alcornocales, con una importante reducción de su superficie actual, en todas las comunidades autónomas, excepto la cornisa cantábrica. Los núcleos permanentes estarían localizados en la media montaña de Gredos, Cádiz, Huelva-Sevilla y Girona. Se localizan 631 estaciones con compatibilidad edáfica comparando con el escenario $A_{1}$, disminuyendo por tanto la compatibilidad de las estaciones en un $43.1 \%$.
En el Escenario $A_{2}$, sólo aparecen estaciones compatibles en Extremadura y Andalucía en los grupos 6, 7, 8 y 9 de carácter mesomediterráneo (Fig. 5). Este escenario limitado por la precipitación parece impedir la existencia de estaciones climáticas homólogas en los grupos del 10 al 15. Los enclaves del norte se mantendrían con cambios menores, pero en el resto peninsular el debilitamiento sería notable.

\section{Escenario $A_{3}$.}

Este escenario sigue mostrando la sensibilidad de los alcornocales a las precipitaciones, puesto que también los factores de estacionalidad de las mismas colocarían a la especie fuera de sus límites actuales de existencia en muchas estaciones (Fig. 3). En este sentido, este escenario es muy parecido al $\mathrm{A}_{1}$, salvo que aquí, con 990 estaciones se perdería un $10.8 \%$ más de estaciones meteorológicas con presencia de alcornoque que en el escenario $A_{1}$, debido al cambio estacional y espacial en la distribución de las precipitaciones. En cambio, este escenario conservaría un $63.7 \%$ de las estaciones con presencia de alcornoque que aparecen en el $A_{2}$. Dependiendo de la importancia que se conceda a la distribución de las precipitaciones estacionales entre las estaciones se determinará qué puede suceder con los alcornocales si se implanta un escenario del tipo $A_{3}$. Si la modificación estacional supone una pérdida de precipitación, la estacionalidad implica grandes efectos sobre la especie, como veremos en el caso del Escenario $\mathrm{A}_{4}$; pero si por el contrario, los cambios estacionales producen un incremento de precipitaciones, la presencia de alcornoque dependerá del tipo de suelos y del encharcamiento de los mismos (Benito et al. 2008, Urbieta et al. 2008). 

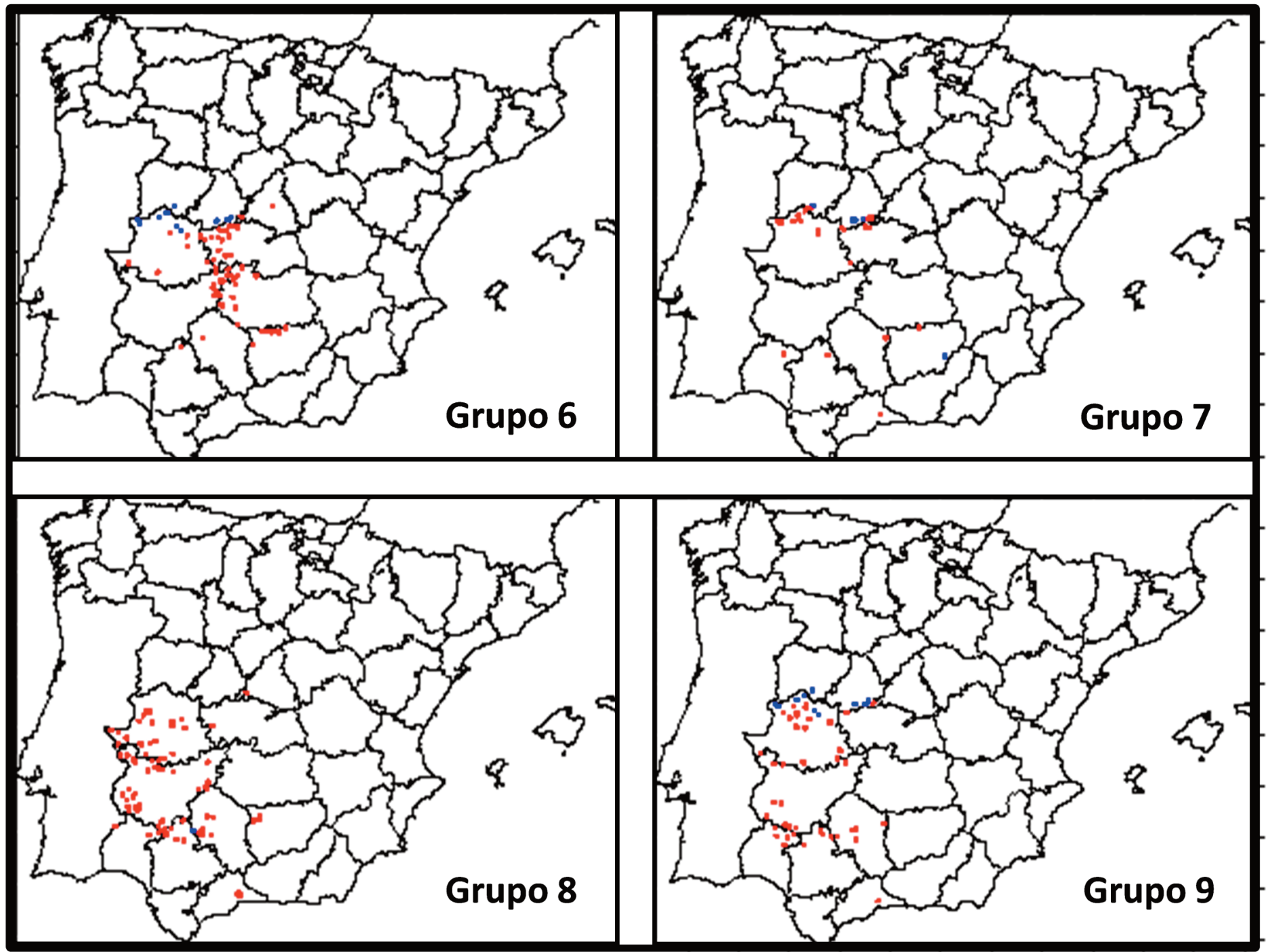

Figura 5. Nuevas posiciones de cada uno de los grupos fitoclimáticos suroccidentales viables para el escenario $A_{2}$. En rojo se ha representado los puntos sin efectos del escenario y en azul la dispersión de nuevas posiciones territoriales en España. Se han escogido estos grupos por la magnitud del impacto en zonas de alto valor actual en la distribución de los alcornocales

En este escenario la distribución espacial del alcornoque seguiría un patrón parecido al escenario $A_{1}$, aunque con una reducción algo mayor de superficie.

Analizando los efectos de este escenario por grupos fitoclimáticos, sólo aparecen en el sur estaciones compatibles en los grupos $6,7,8$ y 9 , de carácter mesomediterráneo. En este escenario, con exceso de precipitación, tampoco aparecerían estaciones climáticas homólogas en los grupos del 10 al 15, de modo que se refleja la gran sensibilidad de los alcornocales ante cualquier modificación del patrón de precipitación, provocando una clara disminución de la potencialidad de las estaciones andaluzas (Fig. 6). El mantenimiento de la elevación térmica limitaría los beneficios del incremento estacional de precipitación.

\section{Escenario $\mathrm{A}_{4}$}

Este escenario es uno de los más limitantes y representa el que parece más probable según las tendencias actuales del clima (IPCC 2007). La pérdida de precipitación media es baja (7\%), pero la estacionalidad está fuertemente alterada por una disminución considerable de precipitaciones primaverales (Fig. 3).

Los alcornocales se desplazarían a las zonas de montaña y se mantendrían en Galicia y la cornisa cantábrica. Los efectos de este escenario son algo mejores a los obtenidos en el escenario $A_{2}$, pero seguirían produciéndose importantes pérdidas superficiales en Andalucía, Extremadura y Cataluña, actuando las áreas montañosas como zonas de reserva para la especie.
De nuevo si analizamos el Escenario $\mathrm{A}_{4}$ por grupos fitoclimáticos, las clases climáticas andaluzas de alcornocal prácticamente desaparecerían. El patrón oriental de precipitación no podría mantener casi ninguna estación homóloga y sólo los grupos 7 y 9 presentarían muy pocas estaciones residuales (Fig. 7).

Un aumento superior a $2^{\circ} \mathrm{C}$ de temperatura en el 2050 no parece probable en esta latitud y con las tendencias actuales y, en todo caso, sería muy improbable que la temperatura media superara los $4^{\circ} \mathrm{C}$. En cualquiera de los escenarios, el incremento térmico de $2^{\circ} \mathrm{C}$ parece lo suficientemente elevado como para que en el futuro se produzcan modificaciones en las estructuras vegetales de nuestro entorno, particularmente en los alcornocales (Benito et al. 2008; Urbieta et al. 2008; Felicísimo et al. 2010).

Por tanto, ante un posible escenario de aumento de temperatura y bajo cualquier patrón de evolución de la precipitación, este trabajo muestra que los alcornocales podrían sufrir un retroceso, pudiendo desplazarse hacia el área eurosiberiana a través del pasadizo ácido de la zona Salamanca-Zamora o a través del territorio portugués según la intersección de estas simulaciones (Fig. 8). Los alcornocales andaluces y extremeños, sobre todo los existentes en el extremo sur, se encontrarían muy comprometidos en todos los casos (Fig. 9 y 10).

\section{¿Qué se puede hacer frente al cambio climático?}

En este trabajo se han estudiado algunos de los posibles impactos más importantes del cambio climático en el alcornoque y se 


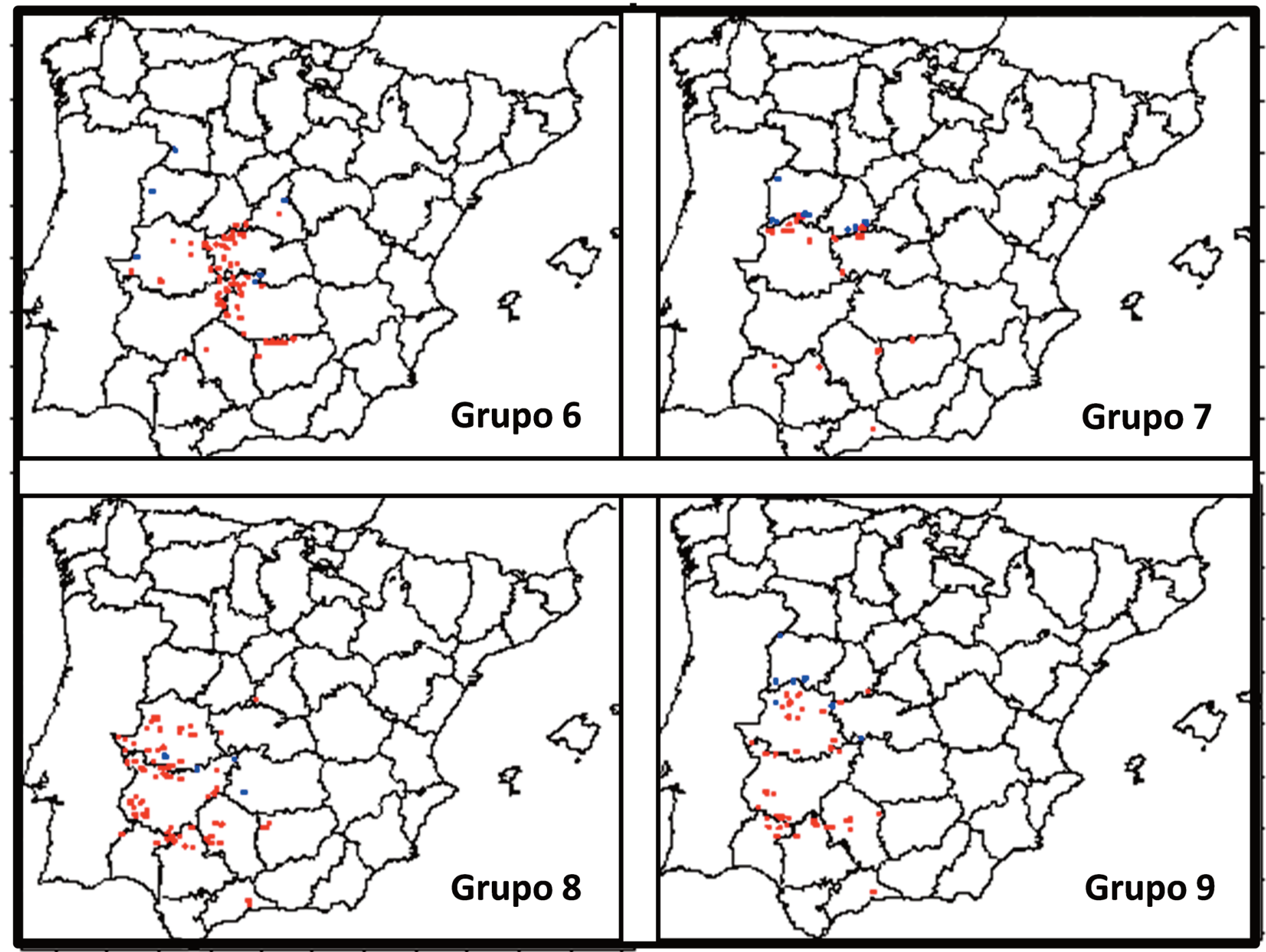

Figura 6. Nuevas posiciones de cada uno de los grupos fitoclimáticos suroccidentales viables en zonas de alto interés para el escenario $A_{3}$. En rojo se ha representado los puntos sin efectos del escenario y en azul la dispersión de nuevas posiciones territoriales en España.

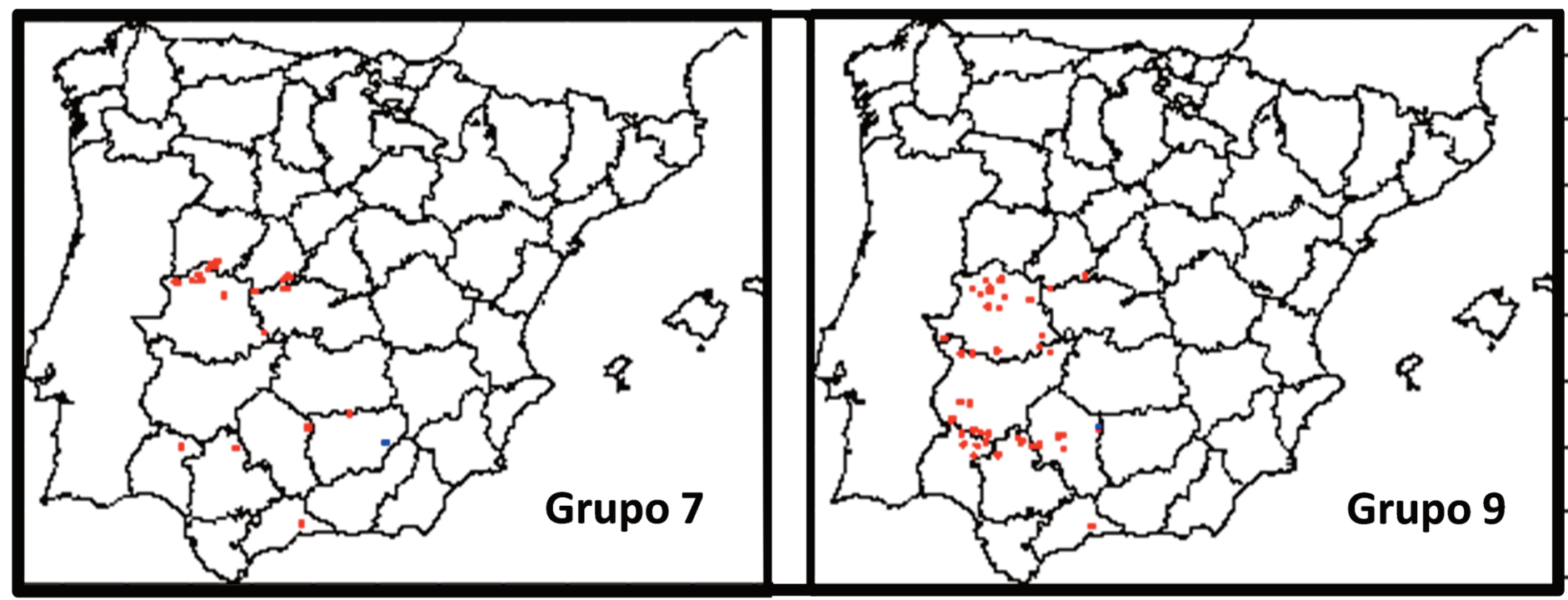

Figura 7. Nuevas posiciones de cada grupo fitoclimático suroccidental viable para el escenario $A_{4}$. En rojo se ha representado los puntos sin efectos del escenario y en azul la dispersión de nuevas posiciones territoriales en España. 


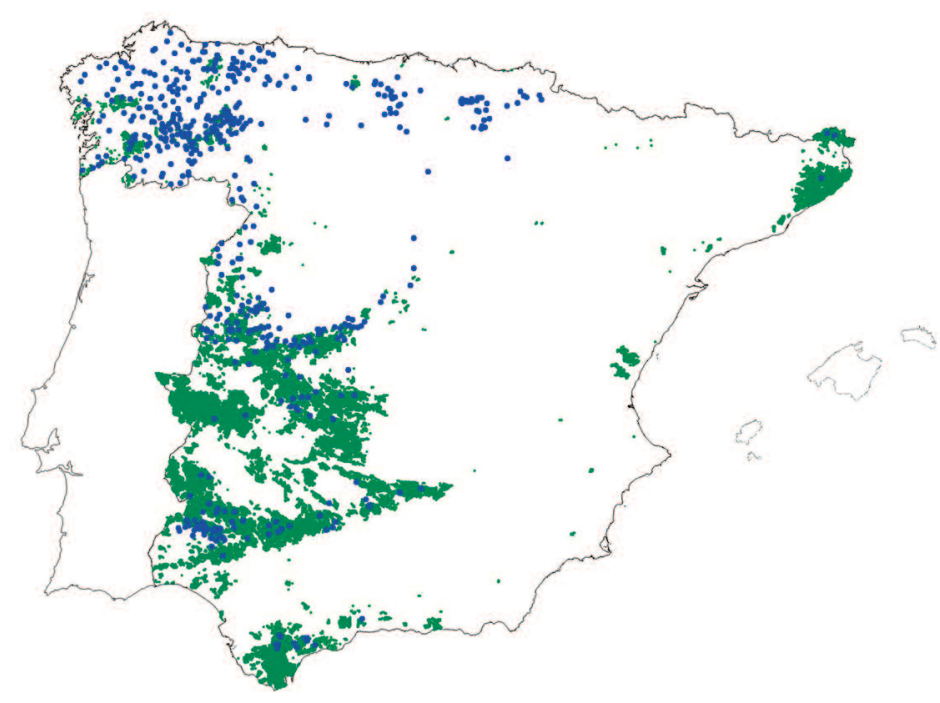

Figura 8. Establecimiento de un escenario de intersección entre los escenarios $A_{1}, A_{2}, A_{3}$ y $A_{4}$.

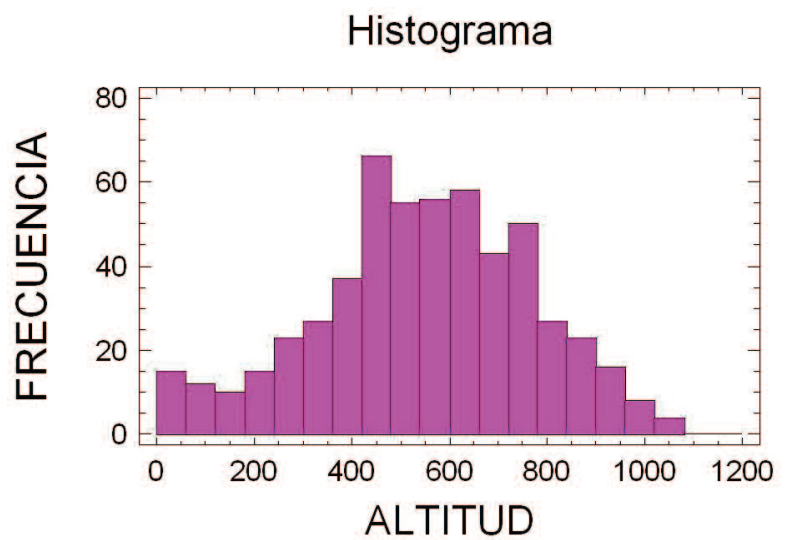

Figura 9. Altitud de los alcornocales en el supuesto de un escenario de refugio.

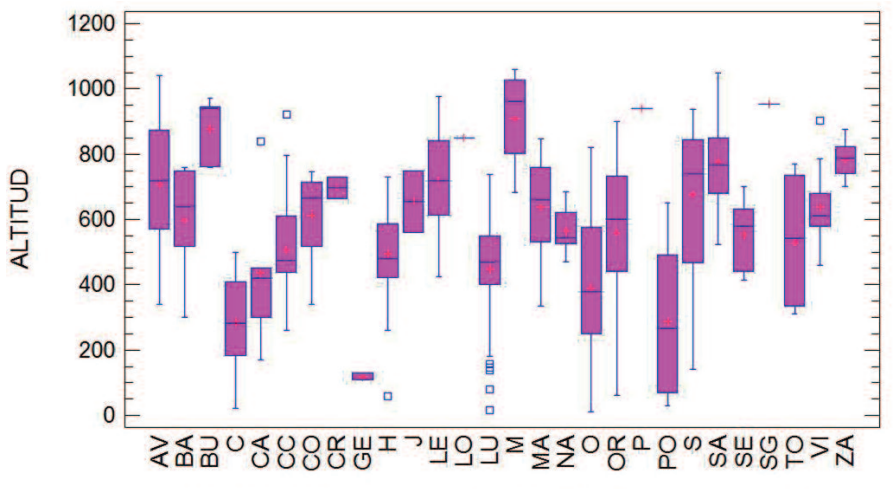

PROVINCIAS EN LAS QUE SE SIMULAN LUGARES DE REFUGIO

Figura 10. Altitud de los lugares de refugio en la intersección de los escenarios $A_{1}, A_{2}, A_{3}$ y $A_{4}$ con respecto a las distintas provincias que los poseen.

ha puesto de manifiesto su vulnerabilidad frente al mismo. También se han sugerido tendencias compatibles con algunos de los efectos que se están produciendo a través de procesos de decaimiento de origen climático (secas) (Fernández-Cancio 1997; 1999; FenándezCancio et al. 2004), concordantes con los modelos que prevén una reducción de esta especie en sus lugares actuales, pudiendo disminuirse las zonas idóneas a mediados del siglo presente en Extremadura y Andalucía y a finales del mismo en Cataluña (Felicísimo et al. 2010). Las nuevas áreas potenciales se localizarían en el Noroeste, Galicia y Asturias occidental, excesivamente lejanas de las actuales para resultar una alternativa planificable en este momento (Benito et al. 2008). Esto hace pensar que es necesario una actuación en la gestión de estos bosques, ya que el alcornoque es una especie con un futuro crítico en una parte importante de su área de distribución, por lo que debería ser objeto de una atención especial en los planes de gestión de ecosistemas ibéricos con presencia principal de especies de Quercus (Fernández-Cancio et al. 2004; Felicísimo et al. 2010).

Al margen de que deban estudiarse más en detalle algunos de estos efectos tanto para el alcornocal como para otros ecosistemas, es urgente diseñar estrategias de mitigación de los efectos del cambio climático y aplicar medidas para incrementar la resistencia y capacidad de adaptación de los ecosistemas frente a los efectos del cambio previsto (Navarro-Cerrillo y Fernández Rebollo 2000; Bronchalo González et al. 2011).

Con base en los resultados de este trabajo y como alternativa a la gestión del ecosistema alcornocal, las nuevas estrategias de mitigación deberían incluir, al menos, las siguientes líneas de actuación:

- Conservar la variabilidad genética de las poblaciones de la especie en la región o Comunidad Autónoma que mayor resiliencia presente a los escenarios previstos de cambio climático, por ejemplo, manteniendo corredores biológicos entre poblaciones viables.

- Diseñar políticas territoriales, incluyendo la ordenación de usos agrícolas y ganaderos, que prevean la distribución futura del alcornocal, considerando los escenarios de cambio climático en el diseño y ejecución de las mismas (Brunet et al. 2009) y las mejoras de los modelos, sobre todo en sus predicciones sobre los patrones de precipitación que hoy poseen una variabilidad excesiva (AEMET 2010; Bronchalo González et al. 2011).

- Asegurar el mantenimiento dentro de la Red de Espacios Naturales Protegidos de representaciones suficientes de las poblaciones y ecosistemas de alcornoque más frágiles. Considerar para ello, la distribución futura del alcornocal, de acuerdo a los escenarios previstos (Montoya 1995; Navarro-Cerrillo et al. 2000)

- Considerar la existencia de refugios fitoclimáticos, entendidos como aquellas zonas que mantendrán cierta estabilidad climática o áreas con climas que en el futuro se considerarán favorables para el alcornoque, definiéndolo en el territorio de cada región o subtipo fitoclimático o Comunidad Autónoma (Fig 9 y 10). Estas zonas deben ser objeto de especial protección pues a partir de ellas pueden establecerse también poblaciones artificiales en esas áreas o áreas alternativas que garanticen la conservación ex situ de la diversidad genética de las especies (Felicísimo et al. 2010).

- Establecer sistemas de seguimiento temporal del cambio en los ecosistemas forestales mediante el establecimiento de Redes de Seguimiento de Bosques o sistemas análogos que han demostrado su viabilidad tanto a nivel europeo, como nacional y autonómico (p.ej., Red Europea, Nacional, Andalucía y Castilla-León) (Dobbertin 2004; Navarro-Cerrillo y Calzado 2004).

- Minimizar el estrés producido por factores no estrictamente climáticos pero que debilitan las masas: por ejemplo reforzar los programas de prevención contra enfermedades y plagas forestales (ver Hódar et al. 2012), en relación con los resultados de las Redes de Equilibrio Biológicos regionales (Navarro-Cerrillo y Calzado, 2001; Navarro-Cerrillo et al. 2001a,b)

- Desarrollar estudios específicos para establecer criterios selvícolas claros que sirvan para mitigar el impacto del cambio climático en los ecosistemas forestales y diseñar una gestión adaptativa. Esto es particularmente importante en el caso de las masas artificiales que pueden ser muy sensibles a un incremento de las situaciones de estrés hídrico debido a la estructura de este tipo de masas (Bronchalo González et al. 2011; Camarero et al. 2012; Candel-Pérez et al. 2012; Linares et al. 2012; Martínez-Vilalta et al. 2012; Sánchez-Salguero et al. 2012a, b). En el caso del alcornoque esta gestión adaptativa debe prestar especial cuidado al proceso de descorche y aprovechamiento (Montero y Cañellas 2003).

- Estudiar los cambios de vegetación actuales para predecir los modelos de composición específica de las diferentes áreas de distribución. 
- Desarrollar modelos predictivos del comportamiento de sistemas productivos de alta singularidad, en particular las dehesas y sistemas agrosilvopastorales afines, para conocer los posibles impactos económicos y ambientales del cambio climático (Bronchalo González et al. 2011).

- Incluir todas estas decisiones en una estrategia nacional de cambio climático, que sea realista, eficaz, dotada de recursos económicos, adecuada, y con actividades concretas que puedan ser fácilmente evaluables y planificadas tanto en el tiempo como en el espacio.

- Y, por supuesto, establecer políticas y estrategias encaminadas a reducir las emisiones de gases de efecto invernadero a la atmósfera, para garantizar imperativamente que la temperatura global no aumente más de $2^{\circ} \mathrm{C}$ respecto a las temperaturas pre-industriales (IPCC 2007).

\section{Conclusiones}

El cambio climático actual se puede estar convirtiendo en un cambio fitoclimático de la vegetación (Fernández-Cancio et al. 2004; 2005, Carnicer et al. 2011; García-López y Allué-Camacho 2011) que podría desplazar los cinturones de vegetación. La variabilidad climática, las sequías y el aumento de temperatura parecen determinantes en la génesis de un fenómeno generalizado.

En este complejo problema hay que considerar la acción conjunta de los agentes bióticos y abióticos, que se entremezclan complementándose en la práctica y sintetizándose en forma de procesos de decaimiento de la vegetación (Fernández-Cancio et al. 2004; Sánchez-Salguero et al. 2012b). La amenaza de generalización del decaimiento forestal es grave e inminente si continúa la alteración del régimen de lluvias y siguen elevándose las temperaturas, según los escenarios climáticos previstos. En nuestros bosques de alcornoque si aumentan los periodos de estrés hídrico, el proceso puede ser brusco y alcanzar límites insospechados e impredecibles en el supuesto de que no funcionen mecanismos de adaptación. El proceso afectará, según los escenarios analizados, especialmente a los bosques de alcornoque de la zona suroccidental de la península. Es posible que en algunas zonas sea incluso necesario realizar sustituciones de las especies principales, sobre todo en el sector suroccidental (alternancia del acebuche en sustitución de alcornoques y encinas, por ejemplo), preservando la biodiversidad autóctona en la medida de lo posible, ya que también algunas especies muy resistentes de estas zonas, como los lentiscos, se están viendo afectadas por decaimientos.

Para identificar las regiones más afectadas se han simulado en este trabajo cuatro escenarios de cambio climático sobre los alcornocales españoles, analizando detalladamente el posible comportamiento futuro. En todos los casos los alcornocales andaluces y catalanes parecen ser los más afectados por los cambios, pero no llegan a desaparecer en ningún supuesto. Las zonas más privilegiadas en Andalucía para su mantenimiento serían Sierra Morena, Sierra de Aracena, Parque Natural de los Alcornocales y puntos con suelos ácidos de la provincia de Málaga siendo el rango altitudinal más favorable para su supervivencia de 400-800 m. Sólo cuando las hipótesis se extreman, los alcornocales desaparecen de Cádiz, pero en muchos escenarios su persistencia parece adquirir un carácter testimonial, tendiendo el monte alcornocal a fragmentarse y desaparecer sin observarse una continuidad territorial.

En la hipótesis de considerar los límites estimados como límites absolutos de existencia del alcornoque en España, según los 15 grupos tipos fitoclimáticos definidos, es muy probable que los escenarios simulados mantengan las masas densas de alcornocal y que las dehesas queden relegadas a las áreas de mayor altitud. Las zonas orientales que permitirían albergar alcornocales, por unos valores de altitud compatible con la especie, quedarían siempre excluidas por ausencia de precipitación o suelos inapropiados.

Finalmente, si se toman los escenarios analizando grupo a grupo se pueden observar las interrelaciones entre las mismas. Los resultados sobre los escenarios señalan la posibilidad de compartir recursos entre clases climáticas adyacentes o entre individuos, en el horizonte del 2050, siempre nororientales y aseguran la posibilidad de mantenimiento de las clases climáticas $6,7,8$ y 9 siempre que las temperaturas no rebasen el umbral de $2^{\circ} \mathrm{C}$. En el caso de las clases $10,11,12$ y 13 la homologación de grupos antes y después del cambio sólo se produce en el escenario $A_{1}$. Las clases 14 y 15 no se mantienen después del cambio simulado en el horizonte de 2050 en ningún supuesto y es aventurado afirmar si van a desaparecer completamente o no.

\section{Agradecimientos}

Este estudio se ha realizado en el marco del proyecto "Evaluación y Control de daños de Seca en encinar-alcornocal en Andalucía" en colaboración con el Proyecto RTA02-042 INIA "Factores Climáticos que Determinan la Distribución de Taxa Mediterráneos de Interés Forestal. Impactos del cambio climático en su gestión y conservación." (Convenio Consejería de Medio Ambiente-Grupo PAI 360-CIFOR-INIA), de los Proyectos DIVERBOS (CGL201130285-C02-02) y ANASINQUE (PGC2010-RNM-5782), y del Proyecto INIA-RTA (RTA2010-00065-00-00) y programa FPU (AP2007-04747) del Ministerio de Educación para RSS. Agradecemos a los técnicos de la Consejería de Medio Ambiente, y al grupo de sanidad forestal de AMAyA por el apoyo logístico, material y de personal para realizar este estudio.

\section{Referencias}

AEMET (Agencia Estatal de Meteorología). 2010. Red de estaciones meteorológicas de España. Ministerio de Agricultura, Pesca y Alimentación. España.

Allen, CD., Macalady, AK., Chenchouni, H., Bachelet, D., McDowell, N., Vennetier, M., Kitzberger, T., Rigling, A., Breshears, DD., Hogg, EH., Gonzalez, P., Fensham, R., Zhang, Z., Castro, J., Demidova, N., Lim, JH., Allard, G., Running, SW., Semerci, A., Cobb, N. 2010. A global overview of drought and heat-induced tree mortality reveals emerging climate change risks for forests. Forest Ecology and Management 259:660-684.

Allué-Andrade J.L., Fernández Cancio, A., 1993. Estado actual y expectativas de la fitoclimatología forestal aspectos fitológicos y dendrológicos. Actas del I Congreso Forestal Español, Lourizán 14-18 Junio de 1993, pp. 71-85. SECF. Disponible en: http://www.congresoforestal.es/

Allué-Andrade, JL. 1995. El cambio climático y los montes españoles. Cuadernos de la Sociedad Española de Ciencias Forestales 2:35-74

Allué-Andrade, JL. 1990. Atlas fitoclimático de España. Taxonomías. Ministerio de Agricultura, Pesca y Alimentación, Instituto Nacional de Investigaciones Agrarias, Madrid. 221 pp.

Allué, M, Montero, G. 1989. Aportaciones al conocimiento fitoclimático de los alcornocales catalanes (Síntesis). Scientia gerundensis 15:162-178

Andreu, L., Gutiérrez, E., Macias, M., Ribas, M., Bosch, O., Camarero, JJ. 2007. Climate increases regional tree-growth variability in Iberian pine forests. Global Change Biology 13:804-815.

Araujo, MB., Guisan, A. 2006. Five (or so) challenges for species distribution modelling. Journal of Biogeography 33:1677-1688.

Benito M., Sánchez de Dios, R., Sainz-Ollero, H. 2008. Effects of climate change on the distribution of Iberian tree species. Applied Vegetation Science 11(2):169-178.

Blanco Castro, E., Casado González, MA, Costa Tenorio, M., Escribano Bombín, R., García Antón, M., Génova Fuster, M., Gómez Manzaneque, A., Gómez Manzaneque, F., Moreno Saiz, JC., Morla Juaristi, C., Regato Pajares, P., Sainz Ollero, H. 1997. Los bosques Ibéricos. Una interpretación geobotánica. Ed. Planeta. Barcelona, España. 572 pp.

Box, EO. 1995. Factors determining distribution of tree species and plant functional types. Vegetatio 121:101-116.

Brasier, CM. 1996. Phytophthora cinnamomi and oak decline in southern Europe. Environmental constraints including climate change. Annals of Forest Science 53:347-358.

Bronchalo González, E., Gutiérrez Teira, A., Picatoste Ruggeroni, JR., Sánchez Peña, G. 2011. Impactos, vulnerabilidad y adpatación al cambio climático en el sector forestal. Ministerio de Medio Ambiente y Medio Rural y Marino. Madrid. España. 126 pp. 
Brunet, M., Casado, MJ., de Castro, M., Galán, P., López, JA. Martín, JM., Pastor, A., Petisco, E., Ramos, P., Ribalaygua, J., Rodríguez, E., Sanz, I., Torres, L. 2009. Generación de escenarios regionalizados de cambio climático para España. AEMET. Ministerio de Medio Ambiente, Medio Rural y Marino. Madrid. España. 165 pp.

Camarero, JJ., Corcuera, L., Peñuelas, J., Gil-Pelegrín, E. 2004. Cambio global y decaimiento del bosque. En: Valladares, F. (Ed.), Ecología del Bosque Mediterráneo en un Mundo Cambiante, pp. 397-423. Ministerio de Medio Ambiente, Madrid, España.

Camarero, JJ., Sangüesa Barreda, G., Alla, AQ., González de Andrés, E., Maestro Martínez, M., Vicente-Serrano, SM. 2012. Los precedentes y las respuestas de los árboles a sequías extremas revelan los procesos involucrados en el decaimiento de bosques mediterráneos de coníferas. Ecosistemas 21(3):22-30.

Candel-Pérez, D., Lucas-Borja, ME., Linares, JC. 2012. Predicciones del crecimiento en poblaciones de pino laricio (Pinus nigra Arn. ssp. salzmanii) bajo diferentes escenarios futuros de cambio climático. Ecosistemas 21(3):41-49.

Carnicer, J., Coll, M., Ninyerola, M., Pons, X., Sanchez, G., Peñuelas, J. 2011. Widespread crown condition decline, food web disruption, and amplified tree mortality with increased climate change-type drought. Proceedings of the National Academy of Science USA 108:1474-1478.

Dobbertin M. (Ed.) 2004. Estimation of growth and yield. En: Programme Coordinating Centre (ed.). Manual on Methods and Criteria for Harmonized Sampling, Assessment, Monitoring and Analysis of the Effects of Air Pollution on Forests. Federal Research Centre for Forestry and Forest Products, Hamburg. Germany. Disponible en: http://icpforests.net/page/icp-forests-manual

Drobyshev, I., Linderson, H., Sonesson, K. 2007. Relationship Between Crown Condition and Tree Diameter Growth in Southern Swedish Oaks. Environmental Monitoring Assessment 128:61-73.

Elena Rosselló, R. 2004. Las clasificaciones biogeoclimáticas: modelos territoriales necesarios para los estudios ecológicos forestales. Investigación Agraria, Sistemas y Recursos Forestales. (Fuera de serie) 13:63-74.

Elena Rosselló, R., Sánchez-Palomares, O, Tella, G., Allué Andrade, JL. 1990. Clasificación biogeoclimática territorial de España: definición de ecorregiones. Ecología. № extra 1:59-80.

Felicísimo, AM.; Muñoz, J.; Villalba, CJ.; Mateo, RG. 2010. Impactos, vulnerabilidad y adaptación al cambio climático de la flora española. Universidad de Extremadura, Real Jardín Botánico (CSIC), Oficina Española de Cambio Climático. 552 pp.

Fernández Cancio, A. 1997. Naturaleza y Significado de La Seca. Un Período Crítico en Nuestra Historia Forestal. Vida Rural 40:56-58.

Fernández Cancio, A. 1999. El fitoclima y la Seca de los Quercus. Actas Congreso sobre Forestación en las Dehesas, Mérida, 20-22.

Fernández Cancio, A., Sardinero, S., Pereira I., Gil, PM., Manrique, E., 2001. Modificación de los Pisos Bioclimáticos Españoles debido a un conjunto de escenarios previsibles de cambio climático. En: Actas del III Congreso Forestal Español, Granada 25 al 28 de septiembre de 2001, pp. 457-464. SECF. Disponible en: http://www.congresoforestal.es/

Fernández-Cancio, A., Manrique-Menéndez, E., Navarro-Cerrillo, R.M, Fernández-Fernández, R. 2004. Fitoclimatología de las especies mediterráneas de Quercus antes un escenario de cambios en el clima. En: Tuset, J.J. y Sánchez, G. 2004. (eds.). La Seca: El decaimiento de encinas, alcornoques y otros Quercus en España. Naturaleza y Parques Nacionales. Serie Técnica. Ministerio de Medio Ambiente. España.

Fernández-Cancio, A., Navarro-Cerrillo, R.M., Fernández-Fernández, R. Calzado Martínez., Gil Hernández, P. 2005. El cambio climático y la vegetación en España. Foresta 32:12-15.

Fernández Cancio, A., Navarro Cerrillo, RM., Sánchez Salguero, R., Fernández Fernández, R., Manrique Menéndez, E. 2011. Viabilidad fitoclimática de las repoblaciones de pino silvestre (Pinus sylvestris L.) en la Sierra de los Filabres (Almería). Ecosistemas 20(1):124-144.

Gandullo, JM, Sánchez-Palomares, O., Muñoz, LA. 1998. Una nueva clasificación climática para España. Ecología. 12:67-77.

García-López J.M., Allué Camacho, C., 2009. Cambio climático y sus posibles efectos sobre la diversidad y vulnerabilidad de los ecosistemas forestales de Castilla y León. En: Actas $5^{\circ}$ Congreso Forestal Español. Sociedad Española de Ciencias Forestales y Junta de Castilla y León, Ávila, 21 al 25 de septiembre de 2009. SECF. Disponible en: http://www.congresoforestal.es/

García-López, J.M., Allué-Camacho, C. 2011. Effects of climate change on the distribution of Pinus sylvestris L. stands in Spain. A phytoclimatic approach to defining management alternatives. Forest Systems 19(3):329-339.
Gil, P.M. 2009. Caracterización fitoclimática de la especies del género Quercus de interés forestal en la España peninsular. Tesis doctoral. ETSIM. Universidad Politécnica de Madrid. Madrid.

Gonzalo Jiménez, J. 2010. Diagnosis fitoclimática de la España peninsular: hacia un modelo de clasificación funcional de la vegetación y de los ecosistemas peninsulares españoles. Naturaleza y Parques $\mathrm{Na}-$ cionales: Serie técnica. Organismo Autónomo Parques Nacionales, Ministerio de Medio Ambiente y Medio Rural y Marino. 441 pp. Madrid, España

Guisan, A., Zimmermann, N.E. 2000. Predictive habitat distribution models in ecology. Ecological Modelling 135:147-186.

Hódar, J.A., Zamora, R., Cayuela, L. 2012. Cambio climático y plagas: algo más que el clima. Ecosistemas 21(3):73-78.

IPCC 2007. Climate Change 2007. The Physical Science Basis. Cambridge, UK. Cambridge University Press, 996 pp.

Linares, J.C., Pazo Sarria, R., Taïqui, L., Camarero, J.J., Ocho, V., Lechuga, V., Seco, J.I., Viñegla, B., Sangüesa, G., Gilarte, P., Merino, J., Carreira, J.A. 2012. Efectos de las tendencias climáticas y la degradación del hábitat sobre el decaimiento de los cedrales (Cedrus atlantica) del norte de Marruecos. Ecosistemas 21(3):7-14

Linder, M., Maroschek, M., Netherer, S., Kremer, A., Barbati, A., GarcíaGonzálo, J., Seidl, R., Delzon, S., Corona, P., Kolström, M., Lexer, M.J., Marchetti, M. 2010. Climate change impacts, adaptative capacity and vulnerability of European forest ecosystems. Forest Ecology and Management. 259(4):698-709.

Lloret, F. 2012. Vulnerabilidad y resiliencia de los ecosistemas terrestres frente a episodios extremos de sequía. Ecosistemas 21(3):85-90.

Maldonado, J., Benito Garzón, M., Sánchez de Dios, Sáinz, H. 2002. Evolución reciente de las áreas de los bosques esclerófilos ibéricos. Cambios deducidos a partir de la cartografía forestal. En: Charco, J. (Coord.) La regeneración Natural del Bosque Mediterráneo en la Península Ibérica, pp. 217-234. ARBA y DGCONA. Madrid, España.

Martínez-Vilalta, J., Aguadé, D., Banqué, M., Barba, J., Curiel Yuste, J., Galiano, L., Garcia, N., Gómez, M., Hereş, A.M., López, B.C., Lloret, F., Poyatos, R. Retana, J. Sus, O., Vayreda, J., Vilà-Cabrera, A. 2012. Las poblaciones ibéricas de pino albar ante el cambio climático: con la muerte en los talones. Ecosistemas 21(3):15-21.

Ministerio de Agricultura, Pesca y Alimentación 1997. Segundo Inventario Forestal Nacional 1986-1995. Madrid. España.

Montero, G., Cañellas, I. 2003. Selvicultura de los alcornocales en España. Silva Lusitana 11(1):1-19.

Montoya, R. 1995. Red de seguimiento de daños en los montes. Daños originados por la sequía en 1994. Cuadernos de la Sociedad Española de Ciencias Forestales 2:83-97.

Montoya, R., López Arias, M. 1998. La red europea de seguimiento de daños en los bosques (nivel I) España 1987-1996. Organismo Autónomo Parques Nacionales. MMA, Madrid, España.

Nakicenovic, N., Alcamo, J., Davis, G., De Vries H.J.M., Fenhann, J., Gaffin, S., Gregory, K., Grubler, A., Jung, TY., Kram, T., La Rovere, EL., Michaelis, L., Mori, S., Morita, T., Papper, W., Pitcher, H., Price, L., Riahi, K., Roehrl, A., Rogner, H.H., Sankovski, A., Schlesinger, M., Shukla, P., Smith, S., Swart, R., Van Rooijen, S.,Victor, N., Dadi, Z., 2000. Special report on emissions scenarios. Intergovernmental Panel on Climate Change, Cambridge University Press, Cambridge. UK.

Navarro-Cerrillo, R.M., Calzado Martínez, C. 2004. Establecimiento de una red de equilibrios biológicos en ecosistemas con presencia de pinsapo (Abies pinsapo Boiss.) en Andalucia. Pirineos 158-159:107-125.

Navarro Cerrillo, R.M., Fernández Rebollo, P. 2000. El síndrome de la Seca del encinar. Propuesta de solución para el Valle de los Pedroches. Fundación Ricardo Delgado Vizcaíno, Pozoblanco, Córdoba. España. $172 \mathrm{pp}$.

Navarro Cerrillo, R.M., Fernández Rebollo, P., Ruiz Navarro, J.M., Vidiella, A. 2001a. El síndrome de la seca en masas de Quercus spp. en Andalucía. En: Actas del III Congreso Forestal Español, Granada 25 al 28 de septiembre de 2001, 489-496. SECF. Disponible en: http://www.congresoforestal.es/

Navarro Cerrillo, RM., Fernández Rebollo, P.; Ruiz Navarro, JM. 2001b. Efecto del descorche y del desbroce sobre el crecimiento diametral del alcornoque (Quercus suber L.) y las condiciones microclimáticas del suelo. Resultados preliminares. Servicio de Ordenación de los Recursos Forestales. Consejería de Medio Ambiente. Junta de Andalucía. Sevilla. España.

Navarro Cerrillo, R.M., Fernández Rebollo, P., Ruiz Navarro, J.M. 2000. Evaluación de daños producidos por la Seca de especies de género Quercus spp. en Andalucía. Servicio de Ordenación de los Recursos Forestales. Consejería de Medio Ambiente. Junta de Andalucía. Sevilla, España.

Peñuelas, J., Lloret, F., Montoya, R. 2001. Severe drought effects on Mediterranean woody flora. Forest Science 47:214-218. 
Pereira Segador, I. 2002. Relaciones Clima-Vegetación en los Alcornocales (Quercus suber L.) españoles. Tesis doctoral. ETSIM. Universidad Politécnica de Madrid. Madrid. España.

Pereira Segador, I., Fernández-Cancio, A. 2001. Aportación al conocimiento fitoclimático de los distintos tipos de alcornocales españoles. Cuadernos de la Sociedad Española de Ciencias Forestales 12:83-89.

Petit, R.J., Hampe, A., Cheddadi, R. 2005. Climate changes and tree phytogeography in the Mediterranean. Taxon 54:877-885.

Ribalaygua, J., Borén, R., Benito, L., Balairón, L. 1998. A Statistical downscaling Method: Description, Validation and Application: First High Resolution Climate Change Scenarios for Spain. En: Proceedings of the European Conference on Applied Climatology- ECAC II. Viena 22 al 25 septiembre de 1998. Disponible en CD-rom.

Rivas-Martínez, S. 2007. Mapa de series, geoseries y geopermaseries de vegetación de España. Memoria del mapa de vegetación potencial de España. Parte 1. Itinera Geobotánica 17:1-222.

Rivas-Martínez, S. 1987. Memoria del mapa de series de vegetación de España. ICONA, Serie Técnica, Servicio de Publicaciones del Ministerio de Agricultura, Pesca y Alimentación, Madrid. España.
Ruiz de la Torre, J. 2006. Flora mayor. Organismo autónomo parques nacionales. Madrid. España.

Sánchez, M.E., Navarro-Cerrillo, RM., Trapero, A., Fernández, P. 2000b. La "seca" de encinas y alcornoques: una visión histórica. Montes 62:29-39.

Sánchez-Salguero, R., Navarro-Cerrillo, R.M., Camarero, J.J., FernándezCancio, A., Swetnam, T.W., Zavala, M.A. 2012a. Vulnerabilidad frente a la sequía de repoblaciones de dos especies de pinos en su límite meridional en Europa. Ecosistemas 21(3):31-40.

Sánchez-Salguero, R., Navarro-Cerrillo, R.M., Swetnam, T.W., Zavala, M.A. $2012 \mathrm{~b}$. Is drought the main decline factor at the rear edge of Europe? The case of southern Iberian pine plantations. Forest Ecology and Management 271:158-169.

Urbieta, I.R., Zavala, M.A., Marañón, T. 2008. Human and non-human determinants of forest composition in southern Spain: evidence of shifts towards cork oak dominance due to last century management. 2008. Journal of Biogeography 35:1688-1700.

Villanueva, J.A. 1990. El Inventario Forestal Nacional, instrumento básico para el conocimiento de la distribución y características de los montes españoles. Ecología, Fuera de Serie n 1:81-93. 